Farbe sich aber nach gelblich-bräunlich geändert hat. HCl-Behandlung bringt die Farbänderung zum Verschwinden, das verbleibende Kristallgerüst unveränderter Gestalt besteht vermutlich aus Kieselsäure, die kaum schon in den ursprünglichen, leicht wasserlöslichen Kriställchen enthalten war, sondern wohl erst aus der Glasunterlage beim Glühprozeß aufgenommen wurde.

Alle diese ungewöhnlichen Eigenschaften drängen natürlich zu der Frage, was das Röntgen-Diagramm über unsere mysteriösen Kriställchen aussagt. Herr Gubser $^{4}$ hat eine Anzahl von Netzebenenabständen d aus einer Guinier-DE-WolfF-Aufnahme ermittelt. In der ASTM-Kartei der d-Linien ist keine definierte Zuordnung zu einer bekannten Substanz auffindbar, so daß das Verfahren einstweilen nur die Kristallnatur der Substanz bestätigt.
Unsere seltenen, seltsamen „Vögel“ sind also Kristalle, die Natrium-Ionen enthalten, leicht in Wasser löslich sind, eine erhebliche Hitzebeständigkeit aufweisen und mancherlei andere Eigenschaften besitzen, ohne daß man bisher den Anionenbestandteil angeben kann. Sicher könnte mit größerem Aufwand noch mehr über die mysteriösen Vögel aufgeklärt werden, die sich während Jahren in dem - vielleicht anomalen - Dunst über der Farbfilmschicht an der gegenüberliegenden - vielleicht auch anomalen - Glasoberfläche langsam ausgebildet haben. Aber cui bono? Es ist ja nichts in Gefahr, keine Fabrikation, kein Verfahren, nicht einmal die schönen Bilder, die durch diesen Vorgang in keiner Weise gelitten haben. Aber immerhin: wer weiterforschen möchte, dem stehen noch Deckgläser mit den mysteriösen Kriställchen zur Verfügung.

${ }^{4}$ Institut für Kristallographie und Petrographie der E. T. H. Zürich, Prof. Dr. F. Laves.

\section{Ordnungsvorgang in aufgedampften Übergangsmetallschichten}

Von Yoshiniкo Mrzushima und Osamu Ochi

Electrical Communication Laboratory, Musashino-shi, Tokio, Japan

(Z. Naturforschg. 18 a, 252-254 [1963] ; eingeg. am 30. November 1961)

Das Tempern aufgedampfter Metallschichten bewirkt bekanntlich eine Widerstandserniedrigung durch Erholung. In der vorliegenden Arbeit wird über das Verhalten von Nickel- und Eisenschichten berichtet, die im Ultrahochvakuum aufgedampft und stufenweise getempert wurden.

Das zum Aufdampfen benutzte Nickel bzw. Eisen wurde zunächst aus 99,9\% reinen Ausgangsmaterialien an dünne Wolframdrähte elektrolytisch niedergeschlagen, deren Gewichte für vorbestimmte Schichtdicken im voraus geeicht wurden. Die Drähte wurden dann in der Versuchszelle (Abb. 1) eingeschmolzen. Die Vier-Zuführungen-Methode ${ }^{1}$ ermöglicht ein Endvakuum von $10^{-11}$ Torr während des Aufdampfens. Als Meßelektroden dienten dünne Platinfolien, die durch Glanzplatin geschützt wurden.

Nach dem Ausheizen und Entgasen erreichte man $10^{-10}$ Torr; dann wurde die Schicht auf die mit flüssigem Stickstoff gekühlte Glaswand aufgedampft. Die Zelle wurde dann abgeschmolzen und in das Temperungsbad eingetaucht. Somit wurde unter Ausschluß von Luft gearbeitet. Die Aufdampfgeschwindigkeit war $>10^{3} \AA / \mathrm{s}$, um Koagulation zu verhindern, und um möglichst viele Störstellen einzufrieren.

1 Y. Mizushima, J. Phys. Soc., Japan 15, 1614 [1960].

2 Y. Mizushima u. O. Ochi, J. Phys. Soc., Japan 11, 893 [1956].

3 R. Suhrmann u. G. Barth, Z. Phys. 103, 10 [1936].

4 L. M. Clarebrough, M. E. Hargreaves u. G. W. West, Phil. Mag. 1, 528 [1956] ; Acta Met. 8, 797 [1960]. - A. SteEger,
Da die Schichtdicke und die Versuchsbedingung so gewählt wurden, daß die Schicht möglichst wenig Koagulation zeigte (keine Widerstandserhöhung, keine halbleitende Eigenschaft, keine elektronenmikroskopisch nachweisbare Körnchenspalte), wird unten nur der Erholungseffekt in Betracht gezogen.

Die Schichtwiderstandsmessungen erfolgten vor und nach jeder Temperaturerhöhung. Aus den Tangenten der Widerstandskurve wurden die Aktivierungsenergien errechnet und für Nickel in Abb. 2 und für Eisen in Abb. 3 dargestellt. Diese Methode hat den Vorteil, daß die Aktivierungsenergie unabhängig von der Reaktionsordnung zu bestimmen ist ${ }^{2}$. Dies gilt unter der Annahme, daß der spezifische Widerstand der entsprechenden Streuzentren temperaturunabhängig ist. Diese Tatsache ist experimentell bestätigt ${ }^{3}$. Die Neigung der Widerstandskurve gegen die Zeit in log-log-Auftragung gibt nach einer Analyse die scheinbare Reaktionsordnung, die in Abb. 4 dargestellt ist.

Die Diffusion der Zentren im Kupfer nach Strahlenschädigung ist weitgehend diskutiert, und die einzelnen Vorgänge sind ziemlich geklärt worden. Eine wichtige Annahme ist dabei, daß Leerstellen und Zwischengitteratome gleich häufig sind, und daß die Diffusion der Leerstellen bei kubisch flächenzentrierten Metallen viel schneller erfolgt als die der Zwischengitteratome.

Die Gleichhäufigkeit ist bei aufgedampften Schichten nicht immer so erfüllt wie bei Strahlenschädigung und Abschreckkühlung des massiven Metalls. Trotzdem werden die vorliegenden Versuchsergebnisse versuchsweise so gedeutet, wie es unten beschrieben ist.

Es besteht Analogie zwischen massivem $\mathrm{Cu}$ und $\mathrm{Ni}^{4}$, und Edelmetallaufdampfschichten ${ }^{2}$. Im Bereich der tief-

P. Schiller u. H. Kronmüller, Phil. Mag. 5, 853 [1960]; Z. Naturforschg. 15 a, 740 [1960]. - D. Schumacher, W. Schüle u. A. Seeger, Z. Naturforschg. 17 a, 228 [1962]. A. van den Beukel, Physica 27, 603 [1961]. 


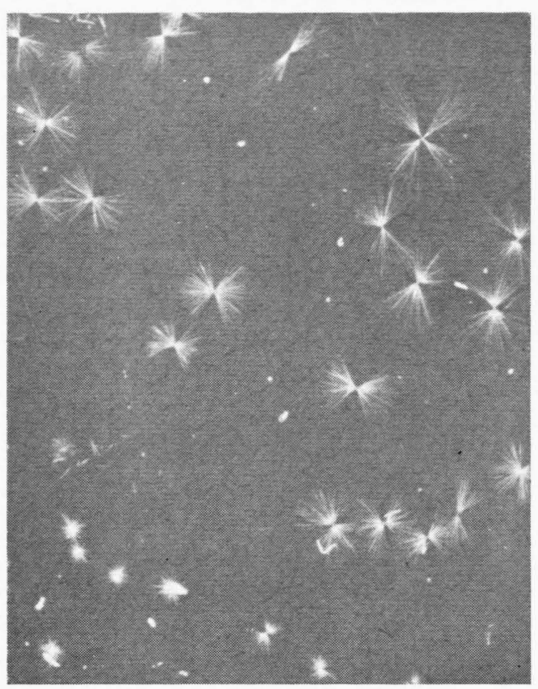

Abb. 1 a. Dunkelfeldaufnahme (Maßstab 20:1) der Zwillingsrosetten des kristallinen Sublimats (Aufn. E. GanseL).

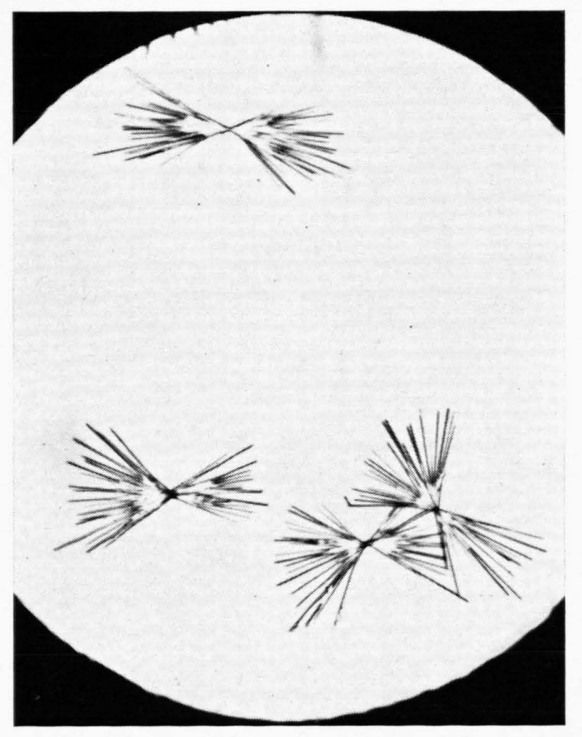

Abb. 1 b. Normale Mikroaufnahme (Maßstab $115: 1$ ) einiger Kristallfächer von a (Aufn. Photographisches Institut der E. T. H. Zürich). 
J. Deмny, Mehrfachkontraste an Versetzungen in Glimmer (S. 261)
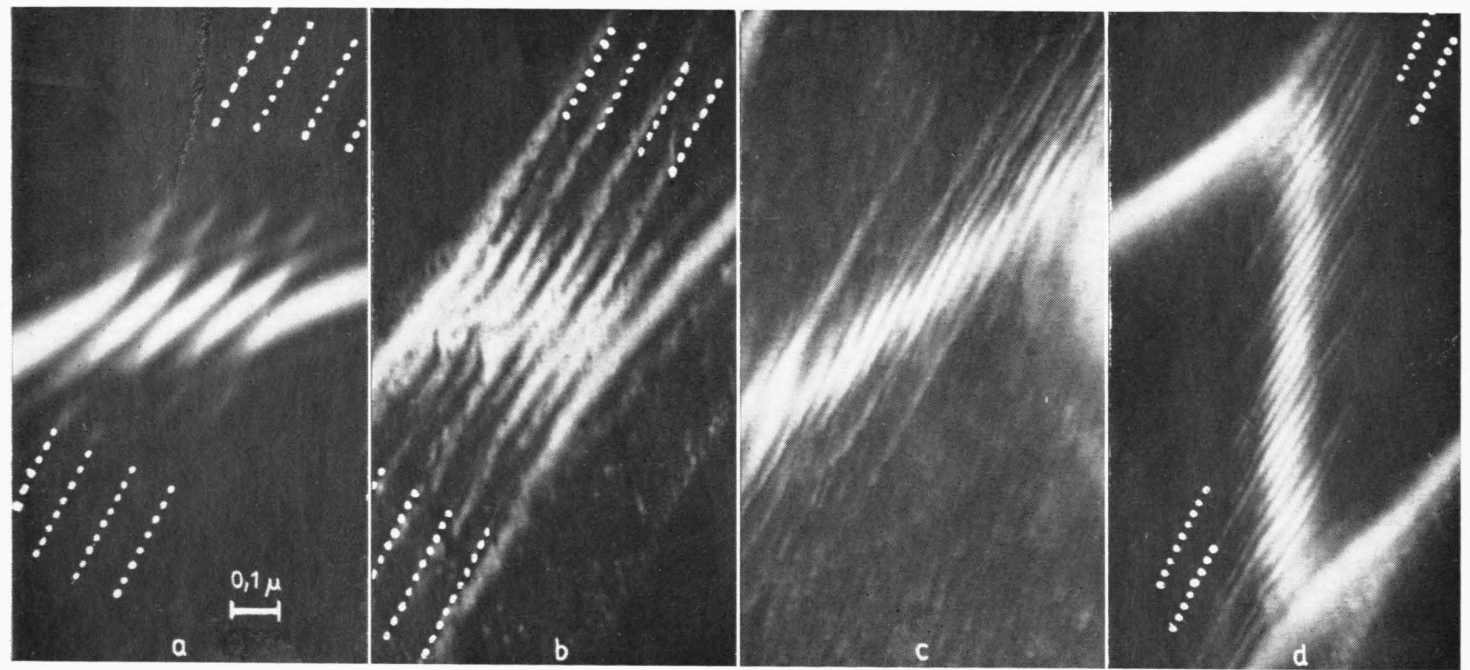

Abb. 2 a-d. Mehrfach-Kontraste an gleichen Versetzungen in Glimmer. Dunkelfeldaufnahmen verschiedener Reflexe,

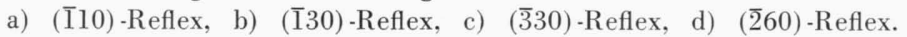



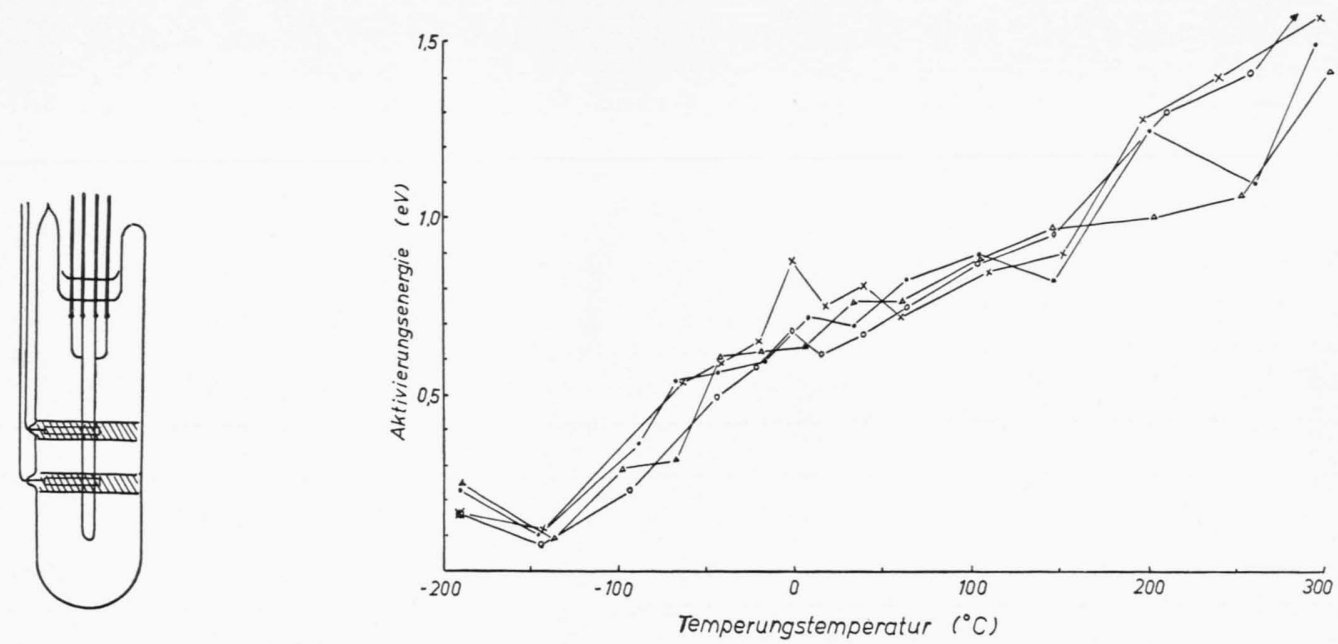

Abb. 1. Schema der Versuchszelle. Die Schicht wird aus Wolframdraht an eine innere Glaswandfläche aufgedampft.
Abb. 3. Aktivierungsenergien des Widerstandes beim Tempern der Eisenschichten.
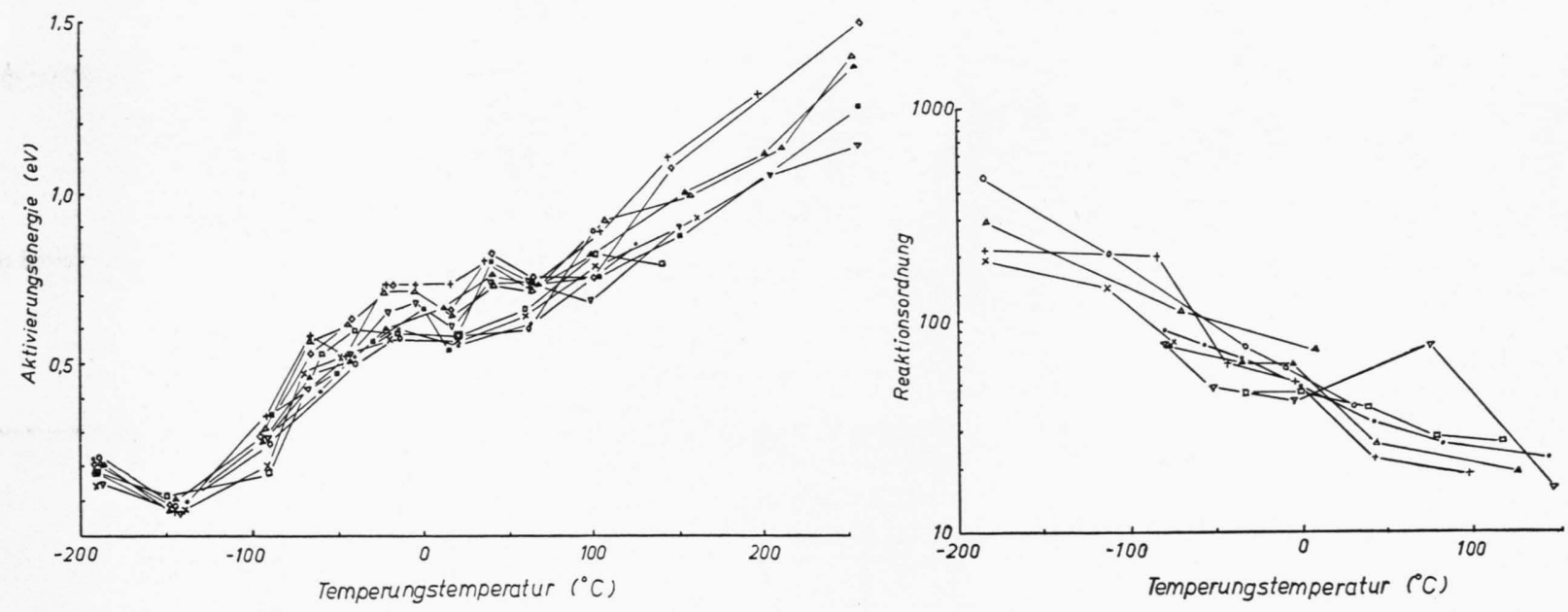

Abb. 2. Aktivierungsenergien des Widerstandes beim Tempern der Nickelschichten. Die Ergebnisse sind unabhängig von der Schichtdicke (100 $\AA$ bis $2000 \AA)$.

sten Temperaturen wird Ausheilen höherer Komplexe und Verschwinden lokaler Spannungen durch Annihilation der Frenket-Paare angenommen. Schon unter Zimmertemperatur setzt auch Diffusion der Zwischengitteratome ein. Über $100{ }^{\circ} \mathrm{C}$ diffundieren die Leerstellen. Die Stufen lassen sich allerdings nicht klar voneinander trennen. Die Rekristallisationstemperatur des Nickels und des Eisens liegt über $500^{\circ} \mathrm{C}$, und Versetzungen können bei der beobachteten Widerstandsänderung vernachlässigt werden, zumal da ihr Einfluß auf den spezifischen Widerstand sehr gering ist.

Eine frisch aufgedampfte Nickelschicht von $100 \AA$ Dicke verliert beim Zimmertemperaturtempern ca. $60 \mu \Omega \mathrm{cm}$ und oberhalb Zimmertemperatur ca. $20 \mu \Omega \mathrm{cm}$
Abb. 4. „Scheinbare“ Reaktionsordnung der Nickelschichten.

(Meßtemperatur $-195{ }^{\circ} \mathrm{C}$ ). Unter der Annahme, daß der spezifische Widerstand des Zwischengitteratoms $2 \mu \Omega \mathrm{cm} / \%^{5} \times($ spezifisches Widerstandsverhältnis $\mathrm{Ni} / \mathrm{Cu}$ $=5$ ) ist, ergibt sich für die Zwischengitteratomdichte ca. 6\%. Die Leerstellendichte ist in gleicher Weise ca. $3 \%$, mit dem spezifischen Widerstand der Leerstelle im Kupfer, $1,5 \mu \Omega \mathrm{cm} / \%$. Diese Werte sind für eine abschreckend kondensierte Schicht nicht zu groß.

Es ist bemerkenswert, daß die Meßkurven für Nickel und Eisen innerhalb der Fehlergrenzen übereinstim-

5 Zum Beispiel R. J. Potter u. D. L. Dexter, Phys. Rev. 108, 677 [1957]. 
men, was zeigt, daß die Diffusionsvorgänge in kubisch flächenzentrierten und kubisch raumzentrierten Metallen gleichartig sind, wahrscheinlich da der Spannungseffekt sehr bedeutend ist.

Die scheinbare Reaktionsordnung wird mit dem Temperungsgang kleiner, doch ist der Wert viel größer als bei einem einfachen Diffusionsvorgang ${ }^{6}$ und als bei Edelmetallschichten ${ }^{2}$. Nach der Deutung von OverhauSER $^{7}$ ist dies auf den mechanischen Spannungseffekt zurückzuführen. Er tritt wegen der Härte des Über-

6 J. Priest, C. Chiou u. H. L. Caswell, J. Appl. Phys. 32, 1772 [1961].

Tunnelwiderstand in Metallkontakten und der Widerstand aufgedampfter Metallschichten

\section{Von Yoshiniko Mizushima und Osamu Ochi}

Electrical Communication Laboratory, Musashino-shi, Tokio, Japan

(Z. Naturforschg. 18 a, 254-255 [1963] ; eingeg. am 12. Dezember 1961)

Holm ${ }^{1}$ hat in seinen Arbeiten über das Kontaktproblem den Tunnelwiderstand dünnster Fremdhäute in Metallkontakten berechnet, wobei er einige vereinfachende Annahmen gemacht hat. Der Tunneleffekt ist von der Temperatur selbstverständlich sehr wenig abhängig und deshalb vernachlässigt. In vorliegender Notiz wird sowohl die Spannungsabhängigkeit als auch die Temperaturabhängigkeit etwas ausführlicher behandelt.

Angenommen wird ein eindimensionales Potentialmodell, wobei ein schmaler Spalt $(S)$ die Kontaktmetalle voneinander trennt (Potentialdifferenz $V$ ). Die Bildkraft für Elektronen läßt sich nach HoLm durch eine Parabel darstellen, wobei am Mittelpunkt $(x=0)$ das höchste Potential herrscht. Die Durchtrittswahrscheinlichkeit $(D)$ ist nach Holm

$$
\begin{aligned}
& D\left(E_{x}\right)=\exp \left[-1,15 \frac{\pi}{2 \hbar} \sqrt{2 m}\left(S-\frac{\varepsilon^{2}}{2 W-E_{x}}\right)\right. \\
& \left.\cdot \sqrt{W-\frac{V}{2}-E_{x}-\frac{\varepsilon^{2}}{S} \ln 2}\right],
\end{aligned}
$$

wobei $\varepsilon$ die elektronische Ladung, $E_{x}$ die Elektronenenergie in $x$-Richtung und $W=\varphi+\zeta$ die innere Austrittsarbeit bedeuten. $\varphi$ ist die äußere Austrittsarbeit und $\zeta$ die Grenzenergie der Elektronen bei $0{ }^{\circ} \mathrm{K}$.

Der Elektronenstrom $(j)$ ergibt sich zu

$$
\begin{aligned}
j=2 \varepsilon\left(\frac{m}{h}\right)^{3} \int_{0}^{\infty} v_{x} D\left(E_{x}\right) \mathrm{d} v_{x} \\
\cdot \iint_{-\infty}^{\infty}[f(E)-f(E+V)] \mathrm{d} v_{y} \mathrm{~d} v_{z},
\end{aligned}
$$

1 R. Holm u. B. Kirschstein, Z. techn. Phys. 16, 488 [1935]. - R. Holm, J. Appl. Phys. 22, 569 [1951]. - R. Holm, Electric Contacts, H. Geber, Stockholm 1946. gangsmetalls auf. Nach der Gleichung von Overhauser ergeben sich Reaktionsordnungen von $10^{2}$ bis $10^{3}$, wenn die Zwischengitteratomdichte etwa $10^{-2}$ beträgt. Dabei errechnet sich die Spannung zu $>10^{9} \mathrm{dyn} / \mathrm{cm}^{2}$. Eine genaue Bestimmung der Aktivierungsenergie des einzelnen Vorganges ohne Spannung ist nicht möglich.

Die Anwesenheit von Sauerstoff während des Aufdampfens bewirkt, daß die Aktivierungsenergie und der Anfangswiderstand größer und der Anteil der Ausheilung kleiner wird ${ }^{6}$.

7 A. W. Overhauser, Phys. Rev. 90, 393 [1953].

wobei $v$ die Elektronengeschwindigkeit und $f(E)$ die FERMische Verteilungsfunktion sind. Das Integral läßt sich umformen ${ }^{1}$

$$
\begin{aligned}
& j=\frac{4 \pi m}{h^{3}} \int_{0}^{\infty} D\left(E_{x}\right) I^{\prime}\left(E_{x}\right) \mathrm{d} E_{x}, \\
& I^{\prime}=\varepsilon \int_{E_{x}}^{\infty}\{f(E)-f(E+V)\} \mathrm{d} E .
\end{aligned}
$$

Für die Auswertung setzt man hierbei

$$
I^{\prime}\left(E_{x}\right)=\varepsilon \bar{V}\left(1-\frac{1}{\beta} \ln \frac{1+e^{\alpha} e^{\beta}}{1+e^{\alpha}}\right),
$$

wobei $\alpha=(E-\zeta) / k T, \quad \beta=V / k T$.

Für jeden Parameter $(T, V, S)$ werden $D(E)$ und $I^{\prime}(E)$ ausgerechnet und das Produkt (Energieverteilungskurve) numerisch integriert. Gold ist als Beispiel angeführt, wobei $\varphi=4,90 \mathrm{eV}$ und $\zeta=5,52 \mathrm{eV}$.

In Abb. 1 ist der Widerstandsverlauf in Abhängigkeit von der Spaltweite gezeigt. Er bezieht sich auf $0{ }^{\circ} \mathrm{K}$ und $V \sim 0$.

Bei einer Spaltweite von $30 \AA$ ist die Spannungsabhängigkeit in Abb. 2 dargestellt. Auch bei Holm liegt ein Widerstandsmaximum bei kleinen Spannungen vor. Er hat das auf sein grobes Potentialmodell zurückgeführt. Das vorliegende Maximum bei besserer Näherung ist etwas kleiner, aber noch deutlich. Wir haben an der unten beschriebenen Metallschicht vergeblich versucht, dieses Maximum experimentell zu finden.

In Abb. 3 ist die Temperaturabhängigkeit bei normaler Temperatur wiedergegeben. Eine halbleitende Eigenschaft ist vorhanden, mit einer Aktivierungsenergie von $1 / 10$ bis $1 / 1000 \mathrm{eV}$. Sie nimmt mit der Spaltweite etwas zu.

Sehr dünne aufgedampfte Metallschichten (z. B. $<100 \AA$ ) zeigen nichtohmische, halbleitende Eigenschaften ${ }^{2}$. Der bisher angenommene Grund ist der Kontaktwiderstand zwischen feinkörnigen Mikrokristal-

2 Zum Beispiel R. Suhrmann u. W. Berndt, Z. Phys. 115, 17 [1940]. 